\title{
Estimativa de entropia de Muscina stabulans (Fallén) (Diptera, Muscidae) em condições artificiais ${ }^{1}$
}

\author{
Rodrigo Ferreira Krüger ${ }^{2,3} \&$ Simone Giehl Erthal ${ }^{4}$
}

${ }^{1}$ Contribuição $N^{\circ} 1561$ do Depto. de Zoologia da Universidade Federal do Paraná (UFPR).

${ }^{2}$ Depto. de Zoologia e Genética, IB, UFPel, Caixa Postal 354, 96010-900, Pelotas, RS, Brasil. rfkruger1974@ yahoo.com.br.

${ }^{3}$ Programa de Pós-Graduação em Ciências Biológicas/Entomologia, Depto. de Zoologia, UFPR.

${ }^{4}$ Centro Educacional de Jovens e Adultos Donizete Romualdo da Silva, Rua Serra Azul, 2428, Centro, 78983-000 Espigão do Oeste-RO, Brasil.

\begin{abstract}
Entropy estimation in Muscina stabulans (Fallén) (Diptera, Muscidae) under laboratory conditions. Entropy $(\mathrm{H})$ as a concept in demography was adapted from that of physics to quantify the impact of mortality on life expectancy and to quantitatively demonstrate mortality tendencies in experimental populations. Entropy was estimated for 160 pairs of Muscina stabulans (Fallén, 1817) in a climate-controlled chamber $\left(24.8^{\circ} \mathrm{C} \pm 0.6^{\circ} \mathrm{C}\right.$, relative humidity 70 $-80 \%, 12 \mathrm{~h}$ photophase). Both sexes had $\mathrm{H}$ values intermediate to those theoretically expected $(0.0-0.5)$, showing that the survival curve is rectangular. The age-specific mortality distribution shows that mortality affects adults of this species in two ways. First, mortality is higher for 10 days from pupal emergence. Second, mortality again increases between 20-30 days after emergence. Small differences in mortality rates cause a greater overall impact on the survival of females.
\end{abstract}

KEYWORDS. Life expectancy; mortality; population dynamics; survival.

\begin{abstract}
RESUMO. Estimativa de entropia de Muscina stabulans (Fallén) (Diptera, Muscidae) em condições artificiais. O conceito de entropia $(H)$ foi adaptado da mecânica estatística para a demografia para quantificar o impacto da mortalidade na expectativa de vida e demonstrar quantitativamente a tendência da mortalidade em populações experimentais. Isto foi verificado para 160 casais de Muscina stabulans (Fallén, 1817) mantidos em câmara climatizada a $24,8^{\circ} \mathrm{C} \pm 0,6^{\circ} \mathrm{C}$, umidade relativa do ar entre 70 e $80 \%$ e fotofase de 12 horas. Nestas condições, machos e fêmeas apresentaram valores de $\mathrm{H}$ intermediários aos valores teóricos de $\mathrm{H}=0$ e $\mathrm{H}=0,5$ demonstrando que para esta espécie, a curva de sobrevivência é do tipo retangular. A distribuição da mortalidade por idade específica indicou que a força desse parâmetro age de dois modos sobre os adultos desta espécie. Em um, a mortalidade tem maior força nos intervalos compreendidos entre a emergência dos adultos e o $10^{\circ}$ dia após este processo. No segundo modo, a força de mortalidade é maior entre o $20^{\circ}$ e $30^{\circ}$ dias após a emergência, sendo que pequenas variações na mortalidade causam maior impacto na sobrevivência das fêmeas do que nos machos.
\end{abstract}

PALAVRAS-CHAVE. Expectativa de vida; dinâmica populacional; mortalidade; sobrevivência.

Muscina stabulans (Fallén, 1817) (Diptera, Muscidae) é uma espécie anautógena (Tirone et al. 1996), cosmopolita, com maior freqüência na Região Neotropical (Carvalho 2002). No Brasil foi encontrada na Bahia, Paraná e São Paulo (Carvalho et al. 2002) com preferência por ambientes urbanos (Linhares 1981; Carvalho et al. 1984), importante para a entomologia forense em países subtropicais (Liu \& Greenberg 1989; Centeno et al. 2002) e capaz de transmitir diversos patógenos (Greenberg 1971). Em granjas avícolas, podem reduzir drasticamente as populações de Musca domestica L., 1758 pois suas larvas são predadoras facultativas de terceiro instar (Skidmore 1985).

As larvas de M. stabulans utilizam recursos efêmeros e temporalmente irregulares, com maior velocidade de desenvolvimento a $26^{\circ} \mathrm{C}$ (Mascarini \& Prado 2002). Espécies que utilizam recursos desse tipo servem como modelos para estudos de estrutura e dinâmica populacional (Moura \& Bonatto 1999), que podem ser realizados através das tabelas de vida, amplamente utilizadas pois determinam a probabilidade de mortalidade e sobrevivência por idade específica. Tais modelos podem prever um ponto de partida para questões fundamentais sobre processos populacionais, identificando dois parâmetros demográficos importantes, a distribuição da mortalidade dos indivíduos por idade específica e a taxa de crescimento da população, sugerindo ainda, uma direção para esse crescimento (Carey 1993).

A taxa intrínseca de aumento natural $\left(\mathrm{r}_{\mathrm{m}}\right)$ e a expectativa de vida ( $e_{\mathrm{x}}$ ) são parâmetros cruciais que descrevem a idade média de morte dos indivíduos em uma população (Demetrius 1979) já que a mortalidade não é igual em todas as idades (Krebs 1994) o que leva a uma distribuição de mortes por idade que poderá assumir dois padrões extremos (Demetrius 1978), sendo possível que todos os indivíduos morram com a mesma idade ou que todos os indivíduos possuam a mesma probabilidade de morrer em cada idade (Carey 1993).

A mortalidade pode ser influenciada pela estrutura genética de uma população e as condições ambientais em que essa população vive, podendo alterar a convexidade da curva de sobrevivência (Demetrius 1979) que tem sido geralmente analisada de forma qualitativa (Moura \& Bonatto 1999).

A quantificação dos valores de convexidade desta curva fornece informações úteis sobre a população, como a tendência 
da mortalidade e a força de mortalidade por idade específica (Demetrius 1979). Isso pode ser obtido por meio da utilização do conceito de entropia (H) em demografia (Demetrius, 1978, 1979; Goldman \& Lord 1986).

O conceito de entropia ou informação pode ser interpretado como a medida da heterogeneidade de uma população com respeito à mortalidade em diferentes idades. Este conceito foi adaptado a partir da mecânica estatística e da teoria ergódica que possibilita ainda descrever o desvio da linearidade da variação do número de sobreviventes em função da idade, quantificando assim o impacto da mortalidade sobre a e $e_{x}$,sendo considerado um sumário estatístico tão significativo quanto $r$ (Demetrius 1978), sintetizando a informação contida na curva de sobrevivência (Tuljapurkar 1982).

Quando H é igual a zero, obtém-se a concavidade mais extrema possível (Carey 1993), uma vez que todos os indivíduos morrem exatamente ao mesmo tempo, em estágios mais avançados de vida, o que dá uma forma retangular à curva de sobrevivência (Vaupel 1986). Já para H igual a um, ocorre um decréscimo geométrico na sobrevivência em relação à idade (Carey 1993), o que corresponderia a uma probabilidade constante de mortalidade em cada idade. Para H igual a 0,5 há uma relação linear entre mortalidade e idade (Demetrius 1978).

A força de mortalidade por idade específica pode ser medida através do produto $e_{x} d_{x}$. Esse produto representa a média ponderada da $\mathrm{e}_{\mathrm{x}}$ em idades específicas ou estágios de desenvolvimento. A mortalidade pode ser relacionada com a $\mathrm{e}_{\mathrm{x}}$ em idades específicas por intermédio dos valores deste produto que significa os dias que seriam ganhos na $\mathrm{e}_{\mathrm{x}}$ desta espécie caso essas mortes fossem evitadas (Carey 1993; Goldman \& Lord 1986).

Como observado por Demetrius (1979), os valores de entropia e da força de mortalidade por idade específica podem mudar de acordo com a variabilidade genética da população nos períodos de vida de diferentes indivíduos, mascarando os resultados finais devido à heterogeneidade populacional. Para avaliar a heterogeneidade dos dados relacionados à quantificação das curvas de sobrevivência são necessárias criações de adultos que formem populações independentes.

A dinâmica populacional de M. stabulans tem sido pouco explorada, apesar de sua importância epidemiológica e ecológica. O conceito de entropia foi utilizado neste trabalho para responder três questionamentos relativos a demografia desta espécie. Qual a tendência da curva de sobrevivência de machos e fêmeas? Durante o período de vida de machos e fêmeas, qual ou quais intervalos etários sofrem maior impacto da mortalidade? E, de que forma os resultados obtidos podem contribuir com o entendimento da performance populacional desta espécie?

\section{MATERIALE MÉTODOS}

Foi utilizada uma colônia de M. stabulans mantida em laboratório, em câmara climatizada, a partir de adultos capturados no Campus da Universidade Federal de Pelotas (UFPel) na cidade de Capão do Leão, RS ( $31^{\circ} 45^{\prime} 48^{\prime \prime}$ S e $\left.52^{\circ} 29^{\prime} 02^{\prime \prime} \mathrm{W}\right)$, para obtenção de ovos, larvas e pupas. Os adultos foram acondicionados em gaiolas com $30 \times 30 \times 30 \mathrm{~cm}$ e alimentados com leite em pó, açúcar refinado e farinha de peixe, numa proporção de 2:2:1, além de água em pequenos frascos com espuma de poliestireno cobrindo a superfície do líquido. A dieta e água foram oferecidas ad libitum conforme estabelecido para outras espécies de Muscidae (Ribeiro et al. 2000; Krüger et al. 2003).

Para obtenção das posturas foi oferecida uma dieta composta de farinha de peixe e serragem, numa proporção de 3:2, levando em consideração dietas utilizadas por outros muscídeos (Ribeiro et al., 2000; Krüger et al. 2003), adicionando-se água até torná-la pastosa. Os ovos foram transferidos para recipientes contendo a mesma dieta utilizada para sua obtenção e colocados em funil para coleta de larvas de terceiro instar na fase pós-alimentar. Neste meio, após a eclosão, as larvas foram alimentadas até o estágio de $\mathrm{L}_{3}$. abandonando o funil e caindo em recipiente contendo serragem úmida. As $\mathrm{L}_{3}$ na fase pós-alimentar e as pupas foram transferidas para recipientes de vidro e mantidas até a emergência dos adultos.

A colônia foi mantida durante todo experimento em câmara climatizada com umidade relativa do ar entre $70 \%$ e $80 \%$ e fotofase de 12 horas. A temperatura foi estimada na câmara da seguinte maneira. As temperaturas mínima e máxima foram estimadas às $7 \mathrm{~h}$ de cada dia e a temperatura média da câmara durante o período de fotofase foi estimada em três ocasiões diárias (10h, 13h e 18h), gerando uma média de temperatura para este intervalo. A temperatura média do período experimental foi de $24,8^{\circ} \mathrm{C} \pm 0,6^{\circ} \mathrm{C}$, conforme a média diária das três temperaturas, mínima, máxima e média do período de fotofase, com variação total de $22^{\circ} \mathrm{C}$ a $28^{\circ} \mathrm{C}$ (Tabela I).

Foram mantidas quatro gaiolas, com as mesmas dimensões descritas anteriormente, com 80 indivíduos em cada, sendo 40 casais por gaiola. Para formar os casais, as pupas foram individualizadas em tubos de ensaio com serragem úmida. Após a emergência foi identificado o sexo e posteriormente os casais foram acondicionados em gaiolas, observados diariamente em intervalos de 24 horas, removendo-se as posturas e insetos mortos.

Para o cálculo da estimativa da entropia $(\mathrm{H})$ foram utilizados os seguintes parâmetros, calculados a partir dos dados de

Tabela I. Média $(\chi)$, desvio padrão (DP) e variação $(\Delta)$ da temperatura mínima (Tmin), máxima (Tmax), média do período de fotofase (Tmf) e média diária (Tmd) da câmara climatizada onde foram mantidas as gaiolas de machos e fêmeas de Muscina stabulans (Fallén) durante o período experimental.

\begin{tabular}{ccccc}
\hline & Tmin & Tmax & Tmf & Tmd \\
& \multicolumn{4}{c}{$\left({ }^{\circ} \mathrm{C}\right)$} \\
\hline$\chi$ & 23,8 & 25,0 & 25,7 & 24,8 \\
DP & 0,7 & 0,5 & 0,9 & 0,6 \\
$\Delta$ & $22,0-24,8$ & $24,3-26,3$ & $25,0-28,0$ & $24,0-26,4$ \\
\hline$\Delta$ total & \multicolumn{4}{c}{$22,0-28,0$} \\
\hline
\end{tabular}


sobrevivência em tabela de vida (Carey 1993): x, é a classe etária compreendida em intervalos de cinco dias; $\mathbf{l}_{\mathbf{x}}$, a proporção de sobreviventes em relação ao intervalo x de idade; $d_{x}=l_{x}-l_{x+1}$ fornece a proporção de indivíduos que morreram entre $\mathbf{x}$ e $\mathbf{x}+\mathbf{1} ; \mathbf{q}_{\mathbf{x}}=\mathbf{d}_{\mathbf{x}} / \mathbf{l}_{\mathbf{x}}$, a probabilidade de morte na idade $\mathbf{x}$ e, $\mathbf{p}_{\mathrm{x}}=\mathbf{1 - \mathbf { q } _ { \mathrm { x } }}$, a probabilidade de sobrevivência. A probabilidade média de sobrevivência foi calculada por $\mathbf{L}_{x}=\mathbf{l}_{x}-\left(\mathbf{d}_{x} / 2\right)$ e a esperança de vida (ex) estimada por $\mathbf{T}_{\mathbf{x}} \mathbf{l}_{\mathbf{x}}$.

O número total de dias restantes aos sobreviventes até a idade máxima w, $\mathbf{T}_{\mathbf{x}}$, foi estimado por: $\sum_{y=x}^{w} L_{y}$

Os valores de entropia $(\mathrm{H})$ foram calculados seguindo-se a expressão: $H=\frac{\sum_{x=0}^{w} e_{x} d_{x}}{e_{0}}$ onde $\mathbf{e}_{\mathrm{x}}$ representa a esperança de vida na idade $\mathrm{x} ; \mathbf{d}_{\mathrm{x}}$ a proporção de indivíduos que morreram entre as idades $\mathrm{x}$ e $\mathrm{x}+1 ; \mathbf{e}_{0}$ a esperança de vida na idade inicial e; w idade máxima alcançada pelo último indivíduo sobrevivente (Carey 1993).

As testemunhas que emergiram da criação em laboratório foram depositadas na Coleção de Insetos de Importância Médica e Veterinária, Departamento de Microbiologia e Parasitologia (CDEMP), Universidade Federal de Pelotas.

\section{RESULTADOS EDISCUSSÃO}

As criações tiveram resultados diferentes sob as mesmas condições experimentais devido à variabilidade genética da população de M. stabulans. Esta variação foi sentida nas curvas de sobrevivência para machos e fêmeas das quatro gaiolas bem como nos valores de entropia que estão demonstrados na figura 1 e nos parâmetros necessários para o cálculo da entropia na Tabela II.

Os valores de entropia para machos $(0,251 ; 0,260 ; 0,214 \mathrm{e}$ $0 ; 280)$ e fêmeas $(0,199 ; 0,276 ; 0,225$ e 0,284$)$ para as respectivas gaiolas de $\mathrm{Aa} \mathrm{D}$ (Fig. 1) foram intermediários aos valores teóricos de $\mathrm{H}=0$ e $\mathrm{H}=0,5$. Com exceção a gaiola $\mathrm{A}$, os valores de $\mathrm{H}$ para machos e fêmeas das outras gaiolas foram muito semelhantes, apesar das fêmeas apresentarem valores maiores do que os machos e da $\mathrm{e}_{0} \mathrm{e} \mathrm{w}$ diferir bastante nestas condições (Tabela II).

Tabela II. Parâmetros utilizados para o cálculo da entropia $(\mathrm{H})$ de machos $\left(\sigma^{\top}\right)$ e fêmeas ( 9 ) de Muscina stabulans (Fallén) a $24,8^{\circ} \mathrm{C} \pm$ $0,6^{\circ} \mathrm{C}$ e umidade relativa do ar entre $70 \%$ e $80 \%$ com fotofase de 12 horas em condições de laboratório. ( $\mathrm{e}_{\mathrm{o}}$ ) esperança de vida na idade inicial, (W) idade máxima em dias alcançada pelo último indivíduo sobrevivente, $\left(\Sigma \mathrm{e}_{\mathrm{x}} \mathrm{d}_{\mathrm{x}}\right)$ soma das médias ponderadas das expectativas de vida em todas as idades.

\begin{tabular}{ccccccccc}
\hline & \multicolumn{2}{c}{ Gaiola A } & \multicolumn{2}{c}{ Gaiola B } & \multicolumn{2}{c}{ Gaiola C } & \multicolumn{2}{c}{ Gaiola D } \\
& $\sigma^{x}$ & + & $\sigma^{x}$ & + & $\sigma^{x}$ & & $\sigma^{x}$ & \\
\hline $\mathrm{e}_{\mathrm{o}}$ & 28,925 & 36,975 & 21,875 & 29,325 & 23,900 & 26,400 & 20,550 & 23,225 \\
$\mathrm{~W}$ & 47 & 55 & 40 & 46 & 35 & 36 & 32 & 35 \\
$\Sigma \mathrm{e}_{\mathrm{x}} \mathrm{d}_{\mathrm{x}}$ & 7,260 & 7,359 & 5,692 & 8,086 & 5,113 & 5,929 & 5,781 & 6,603 \\
\hline
\end{tabular}
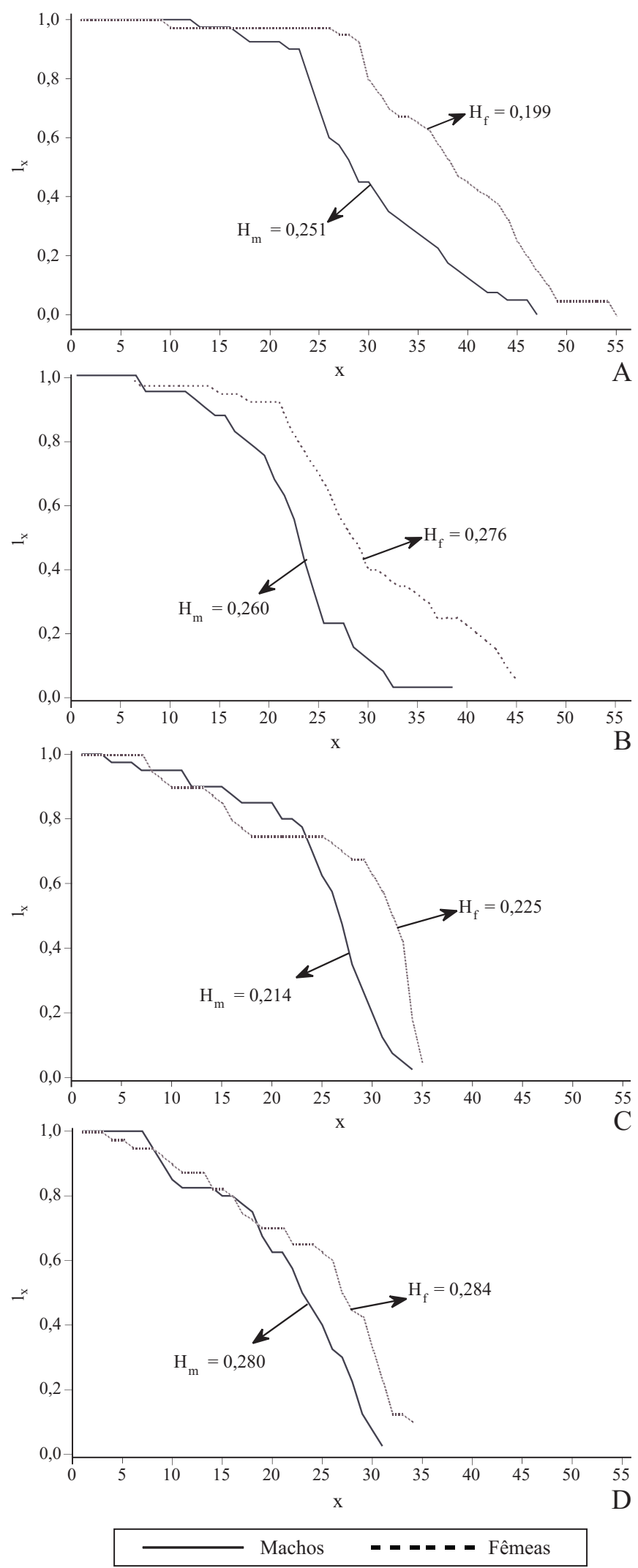

Fig. 1. Curva de sobrevivência de machos e fêmeas de Muscina stabulans (Fallén) a $24,8^{\circ} \mathrm{C} \pm 0,6^{\circ} \mathrm{C}$ e umidade relativa do ar entre $70 \%$ e $80 \%$ com fotofase de 12 horas em condições de laboratório. A, B, C e D gaiolas; $1_{\mathrm{x}}$ - sobrevivência; $\mathrm{x}$ - período em dias; $\mathrm{H}$ - valor de entropia; m - machos; f - fêmeas. 
Nos machos e fêmeas a mortalidade em idades avançadas seguiu um padrão verificado para outras espécies de Diptera. Os valores de entropia (fig. 1) foram semelhantes aos obtidos para Chrysomya megacephala (Fabricius, 1794) (Diptera, Calliphoridae) (Von Zuben et al. 1996), Sarconesia chlorogaster (Wiedemann, 1830) (Calliphoridae) (Moura \& Bonatto 1999) e Lucilia cuprina (Calliphoridae) (Fernandes et al. 2003).

Como a concavidade da curva $1_{x}$ pode ser obtida por intermédio de H (Demetrius 1978), os machos de M. stabulans apresentaram $1_{x}$ levemente mais côncava que a $1_{x}$ das fêmeas (Fig. 1 B,C,D), com exceção da gaiola A, onde ocorreu o inverso (fig. 1A). Para S. chlorogaster e L. cuprina há tendência dos machos possuírem $1_{x}$ levemente mais côncava (Moura \& Bonatto 1999; Fernandes et al. 2003) diferindo do que foi encontrado para C. megacephala (Von Zuben et al. 1996).

Conforme Vaupel (1986), H fornece indícios sobre o efeito da mortalidade por idade específica na e através da mudança percentual produzida por uma redução de $1 \%$ na força de mortalidade em todas as idades. Com isso, uma redução de $1 \%$ na mortalidade de $M$. stabulans, iria mudar a e nos machos de 0,214 a 0,280 dias e nas fêmeas de 0,199 a 0,284 dias sendo semelhante ao que foi obtido para S. chlorogaster (Moura \& Bonatto 1999).

$\mathrm{O}$ efeito da mortalidade em diferentes idades para esta espécie está demonstrado nas Tabelas III e IV através da análise do produto $\mathrm{e}_{\mathrm{x}} \mathrm{d}_{\mathrm{x}}$. Os valores desse produto para machos são maiores do $20^{\circ}$ ao $30^{\circ}, 15^{\circ}$ ao $25^{\circ}, 20^{\circ}$ ao $30^{\circ}$ e do $5^{\circ}$ ao $15^{\circ}$ dias respectivamente para as gaiolas A, B, C e D (Tabela III). Caso as mortes neste período fossem evitadas haveria adição de quatro dias para as gaiolas A e B e cerca de dois a três dias para as gaiolas C e D. Para as fêmeas estes valores foram de quatro, cinco, três e dois dias para as gaiolas A, B, C e D (Tabela IV). Nessas gaiolas, para fêmeas, estes dias que seriam adicionados à $\mathrm{e}_{\mathrm{x}}$, caso estas mortes fossem evitadas, foram compreendidos pelos intervalos do $25^{\circ}$ ao $35^{\circ}, 20^{\circ}$ ao $30^{\circ}, 5^{\circ}$ ao $15^{\circ}$ e de zero ao $10^{\circ}$ dias. Estes períodos são semelhantes entre machos e fêmeas com exceção da gaiola C (Tabelas III e IV).

Tabela III. Valores do produto $\mathrm{e}_{\mathrm{x}} \mathrm{d}_{\mathrm{x}}$ sobre intervalos de cinco dias para machos de Muscina stabulans (Fallén) a $24,8^{\circ} \mathrm{C} \pm 0,6^{\circ} \mathrm{C}$ e umidade relativa do ar entre $70 \%$ e $80 \%$ com fotofase de 12 horas em condições de laboratório. x, classe etária.

\begin{tabular}{ccccc}
\hline & \multicolumn{4}{c}{ Gaiolas } \\
\cline { 2 - 5 } $\mathrm{x}$ & $\mathrm{A}$ & $\mathrm{B}$ & $\mathrm{C}$ & $\mathrm{D}$ \\
\hline 5 & 0,000 & 0,000 & 0,548 & 0,000 \\
10 & 0,000 & 0,794 & 0,486 & 2,491 \\
15 & 0,448 & 0,824 & 1,041 & 0,256 \\
20 & 0,703 & 1,401 & 0,646 & 1,279 \\
25 & 2,769 & 2,024 & 1,051 & 1,264 \\
30 & 1,574 & 0,420 & 1,182 & 0,477 \\
35 & 0,956 & 0,217 & 0,160 & 0,013 \\
40 & 0,635 & 0,013 & - & - \\
45 & 0,150 & - & - & - \\
50 & 0,025 & - & - & - \\
\hline
\end{tabular}

Tabela IV. Valores do produto $\mathrm{e}_{\mathrm{x}} \mathrm{d}_{\mathrm{x}}$ sobre intervalos de cinco dias para fêmeas de Muscina stabulans (Fallén) a $24,8^{\circ} \mathrm{C} \pm 0,6^{\circ} \mathrm{C}$ e umidade relativa do ar entre $70 \%$ e $80 \%$ com fotofase de 12 horas em condições de laboratório. x, classe etária.

\begin{tabular}{ccccc}
\hline & \multicolumn{5}{c}{ Gaiolas } \\
\cline { 2 - 5 } $\mathrm{x}$ & $\mathrm{A}$ & $\mathrm{B}$ & $\mathrm{C}$ & $\mathrm{D}$ \\
\hline 5 & 0,000 & 0,000 & 0,000 & 1,025 \\
10 & 0,733 & 0,608 & 2,031 & 1,254 \\
15 & 0,000 & 0,423 & 1,585 & 0,991 \\
20 & 0,000 & 0,359 & 0,756 & 1,150 \\
25 & 0,000 & 2,829 & 0,183 & 0,689 \\
30 & 2,512 & 2,329 & 0,671 & 1,206 \\
35 & 1,259 & 0,770 & 0,703 & 0,288 \\
40 & 1,435 & 0,490 & - & - \\
45 & 0,900 & 0,277 & - & - \\
50 & 0,321 & - & - & - \\
55 & 0,025 & - & - & - \\
\hline
\end{tabular}

Em geral, uma história de vida ótima é representada pelo balanceamento entre os custos e benefícios de uma mudança na $1_{x}$ estando relacionada ao esforço reprodutivo empreendido pelas fêmeas em uma dada idade específica (Reznick 1985). Conforme haja variação na força de mortalidade por idade específica, poderá haver influência significativa na fecundidade das fêmeas de uma população, caso esta força seja maior em intervalos etários onde haja mais oviposições. Isto foi verificado para Ophyra albuquerquei Lopes, 1986 (Diptera, Muscidae), através de análise qualitativa dos dados de sobrevivência e fecundidade e foi constatado que após sucessivos picos de oviposição ocorreram picos de mortalidade de fêmeas, o que poderia indicar um alto custo fisiológico (Krüger et al. 2004). Este custo se dá, geralmente, nos primeiros dias de uma coorte de uma espécie que tenha alta velocidade de desenvolvimento como é o caso de $M$. stabulans.

Respondendo as questões propostas para este trabalho, machos e fêmeas de M. stabulans apresentam curva de sobrevivência com tendência retangular. Neste caso, a mortalidade tem maior impacto nos primeiros intervalos etários, logo após a emergência dos adultos. Esta força possui dois modos distintos de manifestação nas populações desta espécie nos primeiros dias de vida dos adultos, sendo a variabilidade genética a única resposta possível para este resultado, já que as condições de criação foram iguais para todas as gaiolas.

Mesmo apresentando similaridades quanto à forma da curva de sobrevivência, o impacto da mortalidade é maior nas fêmeas do que nos machos, o que pode alterar os sistemas onde esta espécie é encontrada, conforme preconizado para outras espécies de Diptera (Von Zuben et al. 1993; Moura \& Bonatto 1999).

Estes resultados auxiliam no entendimento da performance populacional de $M$. stabulans, na medida que são as fêmeas os indivíduos responsáveis pela distribuição dos ovos nos substratos de criação das larvas. Logo, se estes indivíduos sofrem maior influência da mortalidade, a probabilidade de localização de novos sítios de criação poderá ser alterada (Roff 
1977), já que entre a emergência dos adultos e os períodos de maior influência da mortalidade, decorre pouco tempo, ressaltando que a mortalidade pode ser influenciada pela própria fecundidade através de custos reprodutivos.

Agradecimentos. A CAPES pela bolsa de doutorado ao primeiro autor e a FAPERGS pela bolsa de iniciação científica concedida ao segundo autor. Ao Dr. Paulo Bretanha Ribeiro pelo empréstimo das instalações do Laboratório de Biologia de Insetos da UFPel. Ao Dr. James Roper pelas correções do abstract.

\section{REFERÊNCIAS}

Carey, J. R. 1993. Applied demography for biologists. New York, Oxford University Press, XVI+260p.

Carvalho, C. J. B. (ed.). 2002. Muscidae (Diptera) of the Neotropical Region: Taxonomy. Curitiba, Ed. UFPR, 287 p.

Carvalho, C. J. B.; J. R. Almeida \& C. B. Jesus. 1984. Dípteros sinantrópicos de Curitiba e arredores (Paraná, Brasil). I. Muscidae. Revista Brasileira de Entomologia 28: 551-560.

Carvalho, C. J. B.; M. O. Moura \& P. B. Ribeiro. 2002. Chave para adultos de dípteros (Muscidae, Fanniidae, Anthomyiidae) associados ao ambiente humano no Brasil. Revista Brasileira de Entomologia 46: 107-114.

Centeno, N.; M. Maldonado \& A. Oliva. 2002. Seasonal patterns of arthropods occurring on sheltered and unsheltered pig carcasses in Buenos Aires Province (Argentina). Forensic International Science 126: 63-70.

Demetrius, L. 1978. Adaptative value, entropy and survivorship curve. Nature 275: 213-214.

Demetrius, L. 1979. Relations between demographic parameters. Demography 16: 329-338.

Fernandes, F. M.; D. M. Lapola; R. Neregato; M. C. Carvalho \& C. J. Von Zuben. 2003. Curva de sobrevivência e estimativa de entropia em Lucilia cuprina (Diptera, Calliphoridae). Iheringia, Ser. Zoologia 93: 319-324.

Goldman, N. \& J. W. Lord. 1986. A new look at entropy and the life table. Demography 23: 275-282.

Greenberg, B. 1971. Flies and disease. Ecology, classification and biotic associations. New Jersey, Princeton University Press, Vol. 1 , VII+586p.

Krebs, C. J. 1994. Ecology: The experimental analysis of distribuition and abundance. New York, Harper Collins College Publishers, XIV+801p.
Krüger, R. F.; P. B. Ribeiro \& C. J. B. Carvalho. 2003. Desenvolvimento de Ophyra albuquerquei Lopes (Diptera, Muscidae) em condições de laboratório. Revista Brasileira de Entomologia 47: 643648.

Krüger, R. F.; P. B. Ribeiro; C. J. B. Carvalho; F. M. Lambrecht \& A. M. Nunes. 2004. Longevidade e oviposição de Ophyra albuquerquei (Diptera, Muscidae) em condições de laboratório. Iheringia, Série Zoologia 94: 211-216.

Linhares, A. X. 1981. Synanthropy of Muscidae, Fanniidae and Anthomyiidae (Diptera) in the city of Campinas, São Paulo, Brazil. Revista Brasileira de Entomologia 25: 231-243.

Mascarini, L. M. \& A. P. Prado. 2002. Thermal Constant of an Experimental Population of Muscina stabulans (Fallén 1817) (Diptera:Muscidae) in the Laboratory. Memórias do Instituto Oswaldo Cruz 97: 281-283.

Moura, M. O. \& S. R. Bonatto. 1999. Análise de sobrevivência e estimativa de entropia para Sarconesia chlorogaster (Wiedemann) (Diptera, Calliphoridae). Revista Brasileira de Zoologia 16: 221-226.

Reznick, D. 1985. Costs of reproduction: an evaluation of the empirical evidence. Oikos 44: 257-267.

Ribeiro, P. B.; C. J. B. Carvalho; P. R. P. Costa \& P. Silveira-Júnior. 2000. Desenvolvimento de Ophyra aenescens Wiedemann, 1830 (Diptera, Muscidae, Azeliinae), em diferentes temperaturas, em condições de laboratório. Revista Brasileira de Agrociência 6: 80-87.

Roff, D. 1977. Dispersal in dipterans: its costs and consequences. Journal of Animal Ecology 46: 443-456.

Skidmore, P. 1985. The biology of the Muscidae of the world. Dordrecht. Dordrecht Kunk. 550 p.

Tirone, G.; P. P. Parise \& R. M. P. Avancini. 1996. Ovarian development in Muscina stabulans (Diptera, Muscidae) on differents diets. Revista Brasileira de Entomologia 40: 105106.

Tuljapurkar, S. D. 1982. Why use population entropy? It determines the rate of convergence. Journal Mathematical of Biology 13: $325-337$.

Vaupel, J. W. 1986. How change in age-specific mortality affects life expectancy. Populations Studies 40: 147-157.

Von Zuben, C. J.; S. F. Reis; J. B. R. Do Val; W. A. C. Godoy \& O. B. Ribeiro. 1993. Dynamics of a mathematical model of Chrysomya megacephala (Diptera, Calliphoridae). Journal of Medical Entomology 30: 443-448.

Von Zuben, C. J.; W. A. C. Godoy \& E. L. A. Monteiro-Filho. 1996. Curva de sobrevivência e estimativa de entropia em Chrysomya megacephala (Diptera, Calliphoridae). Revista Brasileira de Entomologia 40: $221-224$. 